\title{
Three-dimensional Cauchy-Riemann structures and second-order ordinary differential equations
}

\author{
Paweł Nurowski ${ }^{1}$ and George A J Sparling ${ }^{2}$ \\ ${ }^{1}$ Instytut Fizyki Teoretycznej, Uniwersytet Warszawski, ul. Hoza 69, Warszawa, Poland \\ 2 Department of Mathematics, University of Pittsburgh, Pittsburgh, PA, USA \\ E-mail: nurowski@fuw.edu.pl and sparling@twistor.org
}

Received 23 June 2003

Published 17 October 2003

Online at stacks.iop.org/CQG/20/4995

\begin{abstract}
The equivalence problem for second-order ordinary differential equations (ODEs) given modulo point transformations is solved in full analogy with the equivalence problem of nondegenerate three-dimensional Cauchy-Riemann structures. This approach enables an analogue of the Fefferman metrics to be defined. The conformal class of these (split signature) metrics is well defined by each point equivalence class of second-order ODEs. Its conformal curvature is interpreted in terms of the basic point invariants of the corresponding class of ODEs.
\end{abstract}

PACS numbers: $\quad$ 02.30.Fn, 02.30.Hq, 02.40.Dr, 04.20.Cv

\section{Introduction}

This paper aims to explain the relations between two classical geometries: the geometry associated with second-order ordinary differential equations defined modulo point transformations of variables and the geometry of the three-dimensional Cauchy-Riemann (CR) structures.

The geometry associated with second-order ordinary differential equations, considered modulo contact transformations, is trivial-all second-order ODEs are locally contact equivalent to the equation $y^{\prime \prime}=0$. If one considers (more natural) point transformations, all diffeomorphisms of the plane $(x, y)$, then their action on the space of all second-order ODEs has nontrivial orbits- there exist second-order ODEs that are not (even locally) point equivalent. An example of such point inequivalent equations is given by $y^{\prime \prime}=0$ and $y^{\prime \prime}=y^{2}$.

In general, the equation

$$
y^{\prime \prime}=Q\left(x, y, y^{\prime}\right)
$$

with the total differential

$$
D=\partial_{x}+y^{\prime} \partial_{y}+Q \partial_{y^{\prime}}
$$


can be characterized by a number of relative invariants (their vanishing or not is a pointinvariant property of the equation). The two of these invariants of lowest order are

$$
w_{1}=D^{2} Q_{y^{\prime} y^{\prime}}-4 D Q_{y y^{\prime}}-D Q_{y^{\prime} y^{\prime}} Q_{y^{\prime}}+4 Q_{y^{\prime}} Q_{y y^{\prime}}-3 Q_{y^{\prime} y^{\prime}} Q_{y}+6 Q_{y y}
$$

and

$$
w_{2}=Q_{y^{\prime} y^{\prime} y^{\prime} y^{\prime}}
$$

These were known to Lie [18] and used by Tresse [43, 44] in his systematic study of an equivalence problem for second-order ODEs given modulo point transformations. Cartan, in his celebrated paper [4] on projective connections, used the class of second-order ODEs for which the invariant $w_{2}$ vanished as an example of a geometry that naturally gives rise to a Cartan normal projective connection ${ }^{3}$.

The study of the geometry of CR structures was initiated by Poincaré [30], who looked for a higher dimensional generalization of the well-known fact that two real analytic arcs in $\mathbf{C}$ are locally biholomorphically equivalent. Using a heuristic argument, he showed that generic two real three-dimensional hypersurfaces $N_{1}$ and $N_{2}$ embedded in $\mathbb{C}^{2}$ are not, even locally, biholomorphically equivalent. This led Segre [34] to study the equivalence problem for real hypersurfaces of codimension 1 in $\mathbb{C}^{2}$, given modulo the biholomorphisms, a problem which was later solved in full generality by Cartan [5]. Generalization of the problem to $\mathbb{C}^{n}$ with $n>2$ led to the theory of CR structures which is a part of several complex variable theory and lies on the borders between analysis, geometry and studies of PDEs. In this theory a particular role is played by the conformal Fefferman metrics [7] which are Lorentzian metrics naturally defined on a circle bundle over each CR manifold. These metrics were defined by Fefferman in 1976 and, surprisingly, were unnoticed by Cartan in his pioneering paper [5].

The appearance of Lorentzian metrics in the CR-structure theory is not an accident. In the lowest dimension $(n=2)$ this is due to the well-known fact $[32,33,37,39]$ that threedimensional Cauchy-Riemann structures are in one-to-one correspondence with congruences of null geodesics without shear in four-dimensional spacetimes. Many physically interesting spacetimes, such as Minkowski, Schwarzschild, Kerr-Newman, Taub-NUT, Hauser, plane gravitational waves and Robinson-Trautman, etc, admit congruences of such geodesics. The understanding of spacetimes admitting congruences of shear-free and null geodesics from the point of view of the corresponding CR geometry has been quite fruitful in the process of solving Einstein vacuum equations [15-17, 20,38]. In these papers the construction of the solutions of the Einstein equations in terms of the CR functions of the corresponding CR geometry is very much in the spirit of Penrose's twistor theory [27, 28]. More importantly, from quite another point of view, spacetimes admitting shear-free congruences of null geodesics are the Lorentzian analogues of Hermitian geometries in four dimensions. Since Robinson played the crucial role of introducing the shear-free property to general relativity, Trautman has called such manifolds Robinson manifolds [24, 41, 42].

Although it is not immediately self-evident, the geometries associated with second-order ODEs and the three-dimensional CR structures are closely related. This fact was known to Segre who, in this context, was quoted in Cartan's paper [5]. Strangely enough, Cartan in [5] only mentioned that such relations existed but did not spend much time explaining what they were. In addition he does not appear to have used these relations to simplify his approach to the CR structures. In this paper we explain Segre's observation in detail and reconstruct and develop the results of the two Cartan papers $[4,5]$ from this point of view.

3 Cartan's observation has recently been understood from the twistorial point of view in [10] and generalized in [19]. 
Section 2 consists of two parts. The first part contains a review of the concept of a three-dimensional CR structure. This is defined as a natural generalization of the notion of classes of real three-dimensional hypersurfaces embedded, modulo biholomorphisms, in $\mathbb{C}^{2}$. Our definition is much more in the spirit of Cartan's treatment of such hypersurfaces than in the spirit of the modern theory of CR manifolds. This point of view will be adopted throughout the paper. The first part of section 2 ends with the quotation of Cartan's theorem (theorem 1) solving the equivalence problem for three-dimensional CR structures. In the second part of section 2 we give the modern description of this theorem in terms of Cartan's $S U(2,1)$ connection. We also show how Cartan might have used his theorem to associate with each nondegenerate three-dimensional CR structure the Fefferman class of metrics. We analyse the Fefferman metrics using Cartan's normal conformal connection associated with them, and give a new proof, based on the use of Baston-Mason conditions [1], of the fact [14] that these metrics are conformal to Einstein metrics only if the curvature of their CR structure's $S U(2,1)$ connection vanishes. We conclude this section with a formula for the Bach tensor for the Fefferman metrics, expressed in terms of the curvature of the corresponding CR structure's connection.

Section 3, the main section of the paper, explains the analogy between three-dimensional CR structures and second-order ODEs defined modulo point transformations. The basic ingredients of this analogy are given just before definition 3, which states what it means for two second-order ODEs to be point equivalent to each other. Comparison between definitions 2 and 3 makes the analogy self-evident. Using this analogy we are able to formulate theorem 2 which solves the equivalence problem for second-order ODEs given modulo point transformations. By the analogy this theorem is literally the same as theorem 1. The only difference is that now the symbols appearing in the theorem have different interpretations. This new interpretation implies that behind the equivalence problem for second-order ODEs modulo point transformations is a certain Cartan $S L(3, \mathbb{R})$ connection. This fact was, of course, known to Cartan [4], but we are not sure if Cartan would present it in the spirit of our paper even if he had a time machine at his disposal (Cartan's ODE paper [4] dates from 1924, whereas his CR paper [5] is from 1932). After theorem 2 we give a local representation of the point invariants of a second-order ODE obtaining, in particular, Lie's basic relative invariants $w_{1}$ and $w_{2}$. We proceed, exploiting the analogy, to define an ODE analogue of Fefferman metrics, which now have split signature. The conformal class of split signature metrics which is naturally associated with each second-order ODE given modulo point transformations turns out to encode all the point-invariant information about the underlying class of ODEs. In particular, all the Cartan invariants of the point equivalent class of ODEs are derived from the Weyl curvature of the corresponding Fefferman-like metric. These metrics are characterized by proposition 1 and the remark following it, and, some time ago, were considered by one of us (GAJS) within the general framework discussed in [35]. Unlike the CR structure case, there are point equivalent classes of second-order ODEs which have nonvanishing curvature of the Cartan $S L(3, \mathbb{R})$ connection and for which the Fefferman-like metrics are conformal to Einstein metrics. Such metrics may only correspond to the ODEs for which the Lie relative invariants $w_{1}$ and $w_{2}$ satisfy $w_{1} w_{2}=0$ and all of them are presented in the appendix.

Like all the Cartan invariants, the Lie invariants $w_{1}$ and $w_{2}$ are interpreted in terms of the Fefferman-like metrics associated with the class of second-order ODEs that defines them. It turns out that the Fefferman metrics associated with a point equivalence class of secondorder ODEs is always of the algebraic type $N \times N^{\prime}$ in the Cartan-Petrov-Penrose [3, 26, 29] classification of real-valued four-dimensional metrics. This means, in particular, that both the self-dual and the anti-self-dual parts of their Weyl tensor have only one independent component. It turns out that the self-dual part of this tensor is proportional to $w_{1}$ and the 
anti-self-dual part is proportional to $w_{2}$. Thus, the vanishing of one of Lie's relative invariants makes the associated Fefferman-like metric half-flat. This partially explains why, in such cases, these metrics may be conformal to Einstein metrics. The rest of section 3 is devoted to understanding the fact that it is easy to find all second-order ODEs for which $w_{2}=0$, since all of them are of the form

$$
y^{\prime \prime}=A_{0}(x, y)+A_{1}(x, y) y^{\prime}+A_{2}(x, y)\left(y^{\prime}\right)^{2}+A_{3}(x, y)\left(y^{\prime}\right)^{3}
$$

and it is quite difficult to find $Q=Q\left(x, y, y^{\prime}\right)$ for which $w_{1}=0$. From the Fefferman-like metrics point of view the switch between $w_{2}$ and $w_{1}$ is the switch between the self-dual and the anti-self-dual parts of their Weyl tensor. This suggests that invariants $w_{1}$ and $w_{2}$ should be on an equal footing of complexity. To see that this is indeed the case requires another notion of duality - the duality between the point equivalent classes of second-order ODEs. This duality was mentioned by Cartan in [4]. We explain it in detail at the end of section 3 . In particular, in proposition 2, and in the example preceding it, we show how to construct solutions $Q=Q\left(x, y, y^{\prime}\right)$ of $w_{1}=0$ knowing $Q$ which satisfy $w_{2}=0$. The understanding of this duality in terms of the natural double fibration of the first jet bundle associated with the ODE is also given.

Finally, in section 4 we give two applications of the theory presented in sections 2 and 3. The first consists of an algorithm for associating a point equivalence class of second-order ODEs with a given three-dimensional CR structure. This may be of some use in general relativity theory and may provide a new understanding of well-known congruences of shearfree and null geodesics. The second application is, as far as we know, the first example of a large class of split signature 4-metrics which satisfy the Bach equations, are genuinely of algebraic type $N \times N^{\prime}$ and are not conformal to Einstein metrics.

Note on the conventions and the notation. We emphasize that in this paper all our considerations are purely local and concerned with nonsingular points of the introduced constructions. We also mention that, following the old tradition in PDEs, we denote the partial derivatives with respect to the variable associated with index $i$ by the corresponding subscript, for example, $\partial G / \partial z_{i}=G_{i}$.

\section{Three-dimensional CR structures}

A three-dimensional CR structure is a structure which a three-dimensional hypersurface $N$ embedded in $\mathbb{C}^{2}$ acquires from the ambient complex space. Following Cartan [5] this structure can be described in the language of differential forms as follows.

Consider a three-dimensional hypersurface $N$ in $\mathbb{C}^{2}$ defined by means of a real function $G=G\left(z_{1}, z_{2}, \bar{z}_{1}, \bar{z}_{2}\right)$, such that $G_{1} \neq 0$, via

$$
N=\left\{\left(z_{1}, z_{2}\right) \in \mathbb{C}^{2} \mid G\left(z_{1}, z_{2}, \bar{z}_{1}, \bar{z}_{2}\right)=0\right\} .
$$

All information about the structure acquired by $N$ from $\mathbb{C}^{2}$ can be encoded in the two 1-forms

$$
\lambda=i\left(G_{1} \mathrm{~d} z_{1}+G_{2} \mathrm{~d} z_{2}\right) \quad \text { and } \quad \mu=\mathrm{d} z_{2} .
$$

These forms have the following properties:

- $\lambda$ is real, $\mu$ is complex

- $\lambda \wedge \mu \wedge \bar{\mu} \neq 0$ on $N$.

Moreover, if $N$ underlies the biholomorphism

$$
z_{1}=z_{1}\left(z_{1}^{\prime}, z_{2}^{\prime}\right), \quad z_{2}=z_{2}\left(z_{1}^{\prime}, z_{2}^{\prime}\right)
$$


the forms transform according to

$$
\lambda \rightarrow \lambda^{\prime}=a \lambda \quad \text { and } \quad \mu \rightarrow \mu^{\prime}=b \mu+c \lambda,
$$

where $a \neq 0$ (real) and $b \neq 0, c$ (complex) are appropriate functions on $N$. It is easy to see that the vanishing of the 3 -form $\lambda \wedge \mathrm{d} \lambda$ is an invariant property under the biholomorphisms of $\mathbb{C}^{2}$. Thus, the two hypersurfaces

$$
N_{1}=\left\{\left(z_{1}, z_{2}\right) \in \mathbb{C}^{2}: z_{1}-\bar{z}_{1}=0\right\}
$$

and

$$
N_{2}=\left\{\left(z_{1}, z_{2}\right) \in \mathbb{C}^{2}:\left|z_{1}\right|^{2}+\left|z_{2}\right|^{2}-1=0\right\},
$$

with the corresponding forms $\lambda_{1}=i \mathrm{~d} z_{1}$ and $\lambda_{2}=i\left(\bar{z}_{1} \mathrm{~d} z_{1}+\bar{z}_{2} \mathrm{~d} z_{2}\right)$ are not biholomorphically equivalent.

The above considerations motivate the introduction of the following structure on 3-manifolds.

Definition 1. A CR structure $[(\lambda, \mu)]$ on a three-dimensional manifold $N$ is an equivalence class of pairs of 1-forms $(\lambda, \mu)$ such that

- $\lambda$ is real, $\mu$ is complex,

- $\lambda \wedge \mu \wedge \bar{\mu} \neq 0$ on $N$

- two pairs $(\lambda, \mu)$ and $\left(\lambda^{\prime}, \mu^{\prime}\right)$ are in the equivalence relation iff there exist functions $a \neq 0$ (real), $b \neq 0, c$ (complex) on $N$ such that

$$
\lambda^{\prime}=a \lambda, \quad \mu^{\prime}=b \mu+c \lambda, \quad \bar{\mu}^{\prime}=\bar{b} \bar{\mu}+\bar{c} \lambda .
$$

A CR structure is called nondegenerate iff

$$
\mathrm{d} \lambda \wedge \lambda \neq 0
$$

otherwise a CR structure is degenerate.

An obvious class of examples of $\mathrm{CR}$ structures is given by biholomorphically equivalent classes of hypersurfaces in $\mathbb{C}^{2}$. The problem of classifying biholomorphically nonequivalent hypersurfaces in $\mathbb{C}^{2}$ is therefore a part of the equivalence problem of $\mathrm{CR}$ structures.

Definition 2. Let $(N,[(\lambda, \mu)])$ and $\left(N^{\prime},\left[\left(\lambda^{\prime}, \mu^{\prime}\right)\right]\right)$ be two CR structures on two threedimensional manifolds $N$ and $N^{\prime}$. We say that $(N,[(\lambda, \mu)])$ and $\left(N^{\prime},\left[\left(\lambda^{\prime}, \mu^{\prime}\right)\right]\right)$ are (locally) equivalent iff, for any two representatives $(\lambda, \mu) \in[(\lambda, \mu)]$ and $\left(\lambda^{\prime}, \mu^{\prime}\right) \in\left[\left(\lambda^{\prime}, \mu^{\prime}\right)\right]$, there exists a (local) diffeomorphism $\phi: N \rightarrow N^{\prime}$ and functions $a \neq 0$ (real), $b \neq 0, c$ (complex) on $N$ such that

$$
\phi^{*}\left(\lambda^{\prime}\right)=a \lambda, \quad \phi^{*}\left(\mu^{\prime}\right)=b \mu+c \lambda, \quad \phi^{*}\left(\bar{\mu}^{\prime}\right)=\bar{b} \bar{\mu}+\bar{c} \lambda .
$$

It is easy to see that all three-dimensional degenerate $\mathrm{CR}$ structures are locally equivalent to the structure associated with a biholomorphic class of hypersurfaces equivalent to the hypersurface $N_{1}=\mathbb{C} \times \mathbb{R}$. The equivalence problem for nondegenerate three-dimensional CR structures was solved by Cartan [5]. Given a nondegenerate CR-structure $(N,[(\lambda, \mu)])$, he considered the forms

$$
\theta^{1}=b \mu+c \lambda, \quad \theta^{2}=\bar{b} \bar{\mu}+\bar{c} \lambda, \quad \theta^{3}=a \lambda
$$

with some unspecified functions $a \neq 0$ (real), $b \neq 0$ and $c$ (complex). He viewed the forms as being well defined on an eight-dimensional space $P_{0}$ parametrized by the points of $N$ and by the coordinates $(a, b, \bar{b}, c, \bar{c})$. Using his equivalence method (see e.g. [11, 25]) 
he then constructed another eight-dimensional manifold $P$ on which the coframe consisting of the forms $\left(\theta^{1}, \theta^{2}, \theta^{3}\right)$ and the five additional well-defined 1-forms $\left(\Omega_{2}, \bar{\Omega}_{2}, \Omega_{3}, \bar{\Omega}_{3}, \Omega_{4}\right)$ constituted the system of basic biholomorphic invariants of the CR structure. More precisely, he proved the following theorem.

Theorem 1. Every nondegenerate $C R$ structure $(N,[(\lambda, \mu)])$ uniquely defines an eightdimensional manifold $P, 1$-forms $\theta^{1}, \theta^{2}, \theta^{3}, \Omega_{2}, \bar{\Omega}_{2}, \Omega_{3}, \bar{\Omega}_{3}, \Omega_{4}$ and functions $\mathcal{R}, \overline{\mathcal{R}}, \mathcal{S}, \overline{\mathcal{S}}$ on $P$ such that

- $\theta^{1}, \theta^{2}, \theta^{3}$ are as in (3), $\bar{\Omega}_{2}, \bar{\Omega}_{3}$ are respective complex conjugates of $\Omega_{2}, \Omega_{3}$ and $\Omega_{4}$ is real,

- $\theta^{1} \wedge \theta^{2} \wedge \theta^{3} \wedge \Omega_{2} \wedge \bar{\Omega}_{2} \wedge \Omega_{3} \wedge \bar{\Omega}_{3} \wedge \Omega_{4} \neq 0$ at each point of $P$.

The forms satisfy the following equations:

$$
\begin{aligned}
& \mathrm{d} \theta^{1}=\Omega_{2} \wedge \theta^{1}+\Omega_{3} \wedge \theta^{3} \\
& \mathrm{~d} \theta^{2}=\bar{\Omega}_{2} \wedge \theta^{2}+\bar{\Omega}_{3} \wedge \theta^{3} \\
& \mathrm{~d} \theta^{3}=i \theta^{1} \wedge \theta^{2}+\left(\Omega_{2}+\bar{\Omega}_{2}\right) \wedge \theta^{3} \\
& \mathrm{~d} \Omega_{2}=2 i \theta^{1} \wedge \bar{\Omega}_{3}+i \theta^{2} \wedge \Omega_{3}+\Omega_{4} \wedge \theta^{3} \\
& \mathrm{~d} \bar{\Omega}_{2}=-2 i \theta^{2} \wedge \Omega_{3}-i \theta^{1} \wedge \bar{\Omega}_{3}+\Omega_{4} \wedge \theta^{3} \\
& \mathrm{~d} \Omega_{3}=\Omega_{4} \wedge \theta^{1}+\Omega_{3} \wedge \bar{\Omega}_{2}+\mathcal{R} \theta^{2} \wedge \theta^{3} \\
& \mathrm{~d} \bar{\Omega}_{3}=\Omega_{4} \wedge \theta^{2}+\bar{\Omega}_{3} \wedge \Omega_{2}+\overline{\mathcal{R}} \theta^{1} \wedge \theta^{3} \\
& \mathrm{~d} \Omega_{4}=i \Omega_{3} \wedge \bar{\Omega}_{3}+\Omega_{4} \wedge\left(\Omega_{2}+\bar{\Omega}_{2}\right)+\overline{\mathcal{S}} \theta^{1} \wedge \theta^{3}+\mathcal{S} \theta^{2} \wedge \theta^{3} .
\end{aligned}
$$

The functions $\mathcal{R}, \mathcal{S}$, and their respective complex conjugates $\overline{\mathcal{R}}, \overline{\mathcal{S}}$, satisfy

$$
\begin{aligned}
& \mathrm{d} \mathcal{R}=-\mathcal{R}\left(\Omega_{2}+3 \bar{\Omega}_{2}\right)-\mathcal{S} \theta^{1}+\mathcal{R}_{2} \theta^{2}+\mathcal{R}_{3} \theta^{3} \\
& \mathrm{~d} \overline{\mathcal{R}}=-\overline{\mathcal{R}}\left(\bar{\Omega}_{2}+3 \Omega_{2}\right)-\overline{\mathcal{S}} \theta^{2}+\overline{\mathcal{R}}_{2} \theta^{1}+\overline{\mathcal{R}}_{3} \theta^{3}
\end{aligned}
$$

and

$$
\begin{aligned}
& \mathrm{d} \mathcal{S}=-\mathcal{S}\left(2 \Omega_{2}+3 \bar{\Omega}_{2}\right)-i \mathcal{R} \bar{\Omega}_{3}+\mathcal{S}_{1} \theta^{1}+\mathcal{S}_{2} \theta^{2}+\mathcal{S}_{3} \theta^{3} \\
& \mathrm{~d} \overline{\mathcal{S}}=-\overline{\mathcal{S}}\left(2 \bar{\Omega}_{2}+3 \Omega_{2}\right)+i \overline{\mathcal{R}} \Omega_{3}+\overline{\mathcal{S}}_{2} \theta^{1}+\mathcal{S}_{1} \theta^{2}+\overline{\mathcal{S}}_{3} \theta^{3},
\end{aligned}
$$

with appropriate functions $\mathcal{R}_{2}, \mathcal{R}_{3}, \mathcal{S}_{1}, \mathcal{S}_{2}, \mathcal{S}_{3}$ and their conjugates.

The function $\mathcal{S}_{1}$ satisfies

$$
\mathcal{S}_{1}=\overline{\mathcal{S}}_{1}
$$

The above theorem, stated in modern language, means the following. The manifold $P$ is a Cartan bundle $H \rightarrow P \rightarrow N$, with $H$ being a five-dimensional parabolic subgroup of $S U(2,1)$. This latter group preserves the $(2,1)$-signature Hermitian form

$$
h(X, X)=\left(X^{1}, X^{2}, X^{3}\right) \hat{h}\left(\begin{array}{c}
\bar{X}^{1} \\
\bar{X}^{2} \\
\bar{X}^{3}
\end{array}\right), \quad \hat{h}=\left(\begin{array}{ccc}
0 & 0 & 2 i \\
0 & 1 & 0 \\
-2 i & 0 & 0
\end{array}\right) .
$$

The forms $\theta^{1}, \theta^{2}, \theta^{3}, \Omega_{2}, \bar{\Omega}_{2}, \Omega_{3}, \bar{\Omega}_{3}, \Omega_{4}$ of the theorem can be collected into a matrix of 1-forms

$$
\omega=\left(\begin{array}{ccc}
\frac{1}{3}\left(2 \Omega_{2}+\bar{\Omega}_{2}\right) & i \bar{\Omega}_{3} & -\frac{1}{2} \Omega_{4} \\
\theta^{1} & \frac{1}{3}\left(\bar{\Omega}_{2}-\Omega_{2}\right) & -\frac{1}{2} \Omega_{3} \\
2 \theta^{3} & 2 \mathrm{i} \theta^{2} & -\frac{1}{3}\left(2 \bar{\Omega}_{2}+\Omega_{2}\right)
\end{array}\right)
$$


satisfying

$$
\omega \hat{h}+\hat{h} \omega^{\dagger}=0,
$$

which is an $s u(2,1)$-valued Cartan connection [12] on $P$. It follows from equations (4) that the curvature of this connections is

$$
\Omega=\mathrm{d} \omega+\omega \wedge \omega=\left(\begin{array}{ccc}
0 & i \overline{\mathcal{R}} \theta^{1} \wedge \theta^{3} & -\frac{1}{2} \overline{\mathcal{S}} \theta^{1} \wedge \theta^{3}-\frac{1}{2} \mathcal{S} \theta^{2} \wedge \theta^{3} \\
0 & 0 & -\frac{1}{2} \mathcal{R} \theta^{2} \wedge \theta^{3} \\
0 & 0 & 0
\end{array}\right) .
$$

It yields all the invariant information about the corresponding CR structure, very much in the way the Riemann curvature yields all the information about the Riemannian structure.

Remark. Note that the assumption that $\mathcal{R}$ or $\mathcal{S}$ (and, therefore $\overline{\mathcal{R}}$ or $\overline{\mathcal{S}}$ ) is constant on $P$ is compatible with (5) iff $\mathcal{R}=\mathcal{S}=0$ (and, therefore $\overline{\mathcal{R}}=\overline{\mathcal{S}}=0$ ). In such a case the curvature $\Omega$ of the Cartan connection $\omega$ vanishes, and it follows that there is only one, modulo local equivalence, $\mathrm{CR}$ structure with this property. It coincides with the CR structure, which the hypersurface $N_{2}=\mathbf{S}^{3}$ acquires from the ambient space $\mathbb{C}^{2}$ via equations (1) and (2).

Using the matrix elements $\omega_{j}^{i}$ of the Cartan connection $\omega$, it is convenient to consider the bilinear form

$$
G=-i \omega^{3}{ }_{j} \omega^{j}{ }_{1} .
$$

This form, when written explicitly in terms of $\theta^{1}, \theta^{2}, \theta^{3}, \Omega_{2}, \bar{\Omega}_{2}, \Omega_{3}, \bar{\Omega}_{3}, \Omega_{4}$, is given by

$$
G=2 \theta^{1} \theta^{2}+\frac{2}{3 i} \theta^{3}\left(\Omega_{2}-\bar{\Omega}_{2}\right) .
$$

Introducing the basis of vector fields $X_{1}, X_{2}, X_{3}, Y_{2}, \bar{Y}_{2}, Y_{3}, \bar{Y}_{3}, Y_{4}$, the respective dual of $\theta^{1}, \theta^{2}, \theta^{3}, \Omega_{2}, \bar{\Omega}_{2}, \Omega_{3}, \bar{\Omega}_{3}, \Omega_{4}$, one sees that $G$ is a form of signature $(+++-0000)$ with four degenerate directions corresponding to four vector fields $Z_{I}=\left(Y_{2}+\bar{Y}_{2}, Y_{3}, \bar{Y}_{3}, Y_{4}\right)$. These four directions span a four-dimensional distribution which is integrable due to equations (4). Thus, the Cartan bundle $P$ is foliated by four-dimensional leaves tangent to the degenerate directions of $G$. Moreover, equations (4) guarantee that

$$
\mathcal{L}_{Z_{I}} G=A_{I} G,
$$

with certain functions $A_{I}$ on $P$, so that the bilinear form $G$ is preserved up to a scale when Lie transported along the leaves of the foliation. Therefore the four-dimensional space $P / \sim$ of leaves of the foliation is naturally equipped with a conformal class of Lorentzian metrics $\left[g_{F}\right]$, the class to which the bilinear form $G$ naturally descends. The Lorentzian metrics

$$
g_{F}=2 \theta^{1} \theta^{2}+\frac{2}{3 i} \theta^{3}\left(\Omega_{2}-\bar{\Omega}_{2}\right)
$$

on $P / \sim$ coincide with the so-called Fefferman metrics [7] (see also [9]) which Fefferman associated with any nondegenerate CR structure $(N,[(\lambda, \mu)])$.

Introducing the volume form

$$
\eta=\frac{1}{3} \theta^{1} \wedge \theta^{2} \wedge \theta^{3} \wedge\left(\Omega_{2}-\bar{\Omega}_{2}\right)
$$

on $P / \sim$, we observe that the Hodge dualization $*$ of the forms $\theta^{3} \wedge \theta^{1}$ and $\theta^{3} \wedge \theta^{2}$ reads

$$
*\left(\theta^{3} \wedge \theta^{1}\right)=-\frac{1}{i}\left(\theta^{3} \wedge \theta^{1}\right) \quad \text { and } \quad *\left(\theta^{3} \wedge \theta^{2}\right)=\frac{1}{i}\left(\theta^{3} \wedge \theta^{2}\right) .
$$

Thus $\theta^{3} \wedge \theta^{1}$ is self-dual and $\theta^{3} \wedge \theta^{2}$ is anti-self-dual. 
A convenient way of analysing the Fefferman metrics is to look for the Cartan normal conformal connection associated with them. Given a nondegenerate CR structure $(N,[(\lambda, \mu)]$, we define an $s o(4,2)$-valued matrix of 1 -forms $\tilde{\omega}$ on $P$ via

$\tilde{\omega}=$

$\left(\begin{array}{cccccc}\frac{1}{2}\left(\Omega_{2}+\bar{\Omega}_{2}\right) & \frac{i}{2} \bar{\Omega}_{3} & -\frac{i}{2} \Omega_{3} & -\Omega_{4} & \frac{i}{12}\left(\Omega_{2}-\bar{\Omega}_{2}\right) & 0 \\ \theta^{1} & -\frac{1}{3}\left(\Omega_{2}-\bar{\Omega}_{2}\right) & 0 & -\Omega_{3} & \frac{i}{2} \theta^{1} & -\frac{i}{2} \Omega_{3} \\ \theta^{2} & 0 & \frac{1}{3}\left(\Omega_{2}-\bar{\Omega}_{2}\right) & -\bar{\Omega}_{3} & -\frac{i}{2} \theta^{2} & \frac{i}{2} \bar{\Omega}_{3} \\ \theta^{3} & \frac{i}{2} \theta^{2} & -\frac{i}{2} \theta^{1} & -\frac{1}{2}\left(\Omega_{2}+\bar{\Omega}_{2}\right) & 0 & \frac{i}{12}\left(\Omega_{2}-\bar{\Omega}_{2}\right) \\ \frac{1}{3 i}\left(\Omega_{2}-\bar{\Omega}_{2}\right) & \bar{\Omega}_{3} & \Omega_{3} & 0 & \frac{1}{2}\left(\Omega_{2}+\bar{\Omega}_{2}\right) & -\Omega_{4} \\ 0 & \theta^{2} & \theta^{1} & \frac{1}{3 i}\left(\Omega_{2}-\bar{\Omega}_{2}\right) & \theta^{3} & -\frac{1}{2}\left(\Omega_{2}+\bar{\Omega}_{2}\right)\end{array}\right)$.

This is a pullback of the Cartan normal conformal connection associated with the Fefferman metric from the Cartan $S O(4,2)$ conformal bundle to $P$. With a slight abuse of the language we call $\tilde{\omega}$ the Cartan conformal connection. The pulback of the curvature of this connection

$$
\tilde{\Omega}=\mathrm{d} \tilde{\omega}+\tilde{\omega} \wedge \tilde{\omega}
$$

is given by

$$
\begin{aligned}
& \tilde{\Omega}=\tilde{\Omega}^{+}+\tilde{\Omega}^{-} \\
& =\left(\begin{array}{cccccc}
0 & -\frac{i}{2} \overline{\mathcal{R}} & 0 & \overline{\mathcal{S}} & 0 & 0 \\
0 & 0 & 0 & 0 & 0 & 0 \\
0 & 0 & 0 & \overline{\mathcal{R}} & 0 & -\frac{i}{2} \overline{\mathcal{R}} \\
0 & 0 & 0 & 0 & 0 & 0 \\
0 & -\overline{\mathcal{R}} & 0 & 0 & 0 & \overline{\mathcal{S}} \\
0 & 0 & 0 & 0 & 0 & 0
\end{array}\right) \theta^{3} \wedge \theta^{1}+\left(\begin{array}{cccccc}
0 & 0 & \frac{i}{2} \mathcal{R} & \mathcal{S} & 0 & 0 \\
0 & 0 & 0 & \mathcal{R} & 0 & \frac{i}{2} \mathcal{R} \\
0 & 0 & 0 & 0 & 0 & 0 \\
0 & 0 & 0 & 0 & 0 & 0 \\
0 & 0 & -\mathcal{R} & 0 & 0 & \mathcal{S} \\
0 & 0 & 0 & 0 & 0 & 0
\end{array}\right) \theta^{3} \wedge \theta^{2} .
\end{aligned}
$$

Here $\tilde{\Omega}^{+}$and $\tilde{\Omega}^{-}$denote the self-dual and the anti-self-dual parts of $\tilde{\Omega}$, respectively.

The theory of the conformal connections $[8,12,13,21]$ then implies that the Weyl curvature 2-form $C$ of $g_{F}$ is given by ${ }^{4}$

$$
\begin{aligned}
C & =C^{+}+C^{-} \\
& =\overline{\mathcal{R}}\left(\begin{array}{cccc}
0 & 0 & 0 & 0 \\
0 & 0 & 1 & 0 \\
0 & 0 & 0 & 0 \\
-1 & 0 & 0 & 0
\end{array}\right) \theta^{3} \wedge \theta^{1}+\mathcal{R}\left(\begin{array}{cccc}
0 & 0 & 1 & 0 \\
0 & 0 & 0 & 0 \\
0 & 0 & 0 & 0 \\
0 & -1 & 0 & 0
\end{array}\right) \theta^{3} \wedge \theta^{2},
\end{aligned}
$$

i.e. denoting the matrix elements of $\tilde{\Omega}$ by $\tilde{\Omega}^{A}{ }_{B}, A, B=0,1, \ldots, 5$, it is given by $\tilde{\Omega}^{A}{ }_{B}$ with $A, B=1,2,3,4$. The very simple form of the Weyl curvature $C$ shows that the Fefferman metric $g_{F}$ of any nondegenerate CR structure $(N,[(\lambda, \mu)])$ is of Petrov type $N$.

Remark. Note that the curvature of the Cartan normal conformal connection $\tilde{\omega}$ of the Fefferman metric $g_{F}$ yields essentially the same information as the curvature of the $s u(2,1)$ valued connection $\omega$. This is due to the fact [2] that $\omega$ is simply an $s u(2,1)$ reduction of the Cartan normal conformal connection associated with the Fefferman metric $g_{F}$. In addition, this indicates the well-known fact that the Fefferman conformal class of metrics $\left[g_{F}\right]$ associated with a given nondegenerate $\mathrm{CR}$ structure $(N,[(\lambda, \mu)])$ yields all the invariant information

${ }^{4}$ Here $C$ has tensor indices $C^{\mu}{ }_{\nu}, \mu, v=1,2,3,4$, which are associated with the null tetrad $\theta^{1}, \theta^{2}, \theta^{3}, \theta^{4}=$ $\frac{1}{3 i}\left(\Omega_{2}-\bar{\Omega}_{2}\right)$ of $g_{F}$. In this tetrad $g_{F}=2 \theta^{1} \theta^{2}+2 \theta^{3} \theta^{4}$. 
about $(N,[(\lambda, \mu)])$. In particular, the $\operatorname{su}(2,1)$-curvature properties of the CR structure are totally encoded in the Weyl tensor 2-forms $C$ of the corresponding Fefferman metrics. Note that although $C$ explicitly involves only $\mathcal{R}$ and $\overline{\mathcal{R}}$, the $\mathcal{S}$ and $\overline{\mathcal{S}}$ functions can be derived from them by means of equations (5).

It is known [14] that the Fefferman metrics are conformal to the Einstein metrics only in the case when the corresponding CR structure is flat $(\Omega=0)$. To see this we recall the Baston-Mason result [1] stating that there are two necessary conditions for a four-dimensional metric $g=g_{\mu \nu} \theta^{\mu} \theta^{\nu}$ to be conformal to an Einstein metric. These, when expressed in terms of the Cartan normal conformal connection $\tilde{\omega}$, are ${ }^{5}$

$$
\mathrm{d} * \tilde{\Omega}+\tilde{\omega} \wedge * \tilde{\Omega}-* \tilde{\Omega} \wedge \tilde{\omega}=0 \quad \text { and }
$$$$
\text { (ii) } \quad\left[\tilde{\Omega}_{\mu \nu}^{+}, \tilde{\Omega}_{\rho \sigma}^{-}\right]=0 \text {, }
$$

where $\tilde{\Omega}^{ \pm}=\frac{1}{2} \tilde{\Omega}^{ \pm}{ }_{\mu \nu} \theta^{\mu} \wedge \theta^{\nu}$. Note that condition (i) is equivalent to the vanishing of the Bach tensor of $g$.

Calculating $\left[\tilde{\Omega}^{-}{ }_{32}, \tilde{\Omega}^{+}{ }_{31}\right]$ for the Fefferman metrics (8) yields

$$
\left[\tilde{\Omega}^{-}{ }_{32}, \tilde{\Omega}^{+}{ }_{31}\right]=i \mathcal{R} \overline{\mathcal{R}}\left(\begin{array}{cccccc}
0 & 0 & 0 & 1 & 0 & 0 \\
0 & 0 & 0 & 0 & 0 & 0 \\
0 & 0 & 0 & 0 & 0 & 0 \\
0 & 0 & 0 & 0 & 0 & 0 \\
0 & 0 & 0 & 0 & 0 & 1 \\
0 & 0 & 0 & 0 & 0 & 0
\end{array}\right),
$$

so that the above condition (ii) is satisfied iff $\mathcal{R}=0$. This means that the corresponding CR structure is flat. It follows that if $\mathcal{R}=0$ the corresponding Fefferman metrics are conformal to the Minkowski metric. In the nonflat $(\mathcal{R} \neq 0)$ case the Fefferman metrics are not conformal to Einstein metrics. Note also that despite this fact the principal null direction of the Fefferman metrics (which in the notation of (8) is tangent to the vector field dual to the form $\theta^{3}$ ) is geodesic and shear free. It has nonvanishing twist and generates a one-parameter conformal symmetry of $g_{F}$.

We conclude this section with the formula for $\tilde{D} * \tilde{\Omega}=\mathrm{d} * \tilde{\Omega}+\tilde{\omega} \wedge * \tilde{\Omega}-* \tilde{\Omega} \wedge \tilde{\omega}$ which, for the Fefferman metrics (8), reads

$$
\tilde{D} * \tilde{\Omega}=-\frac{2}{i} \mathcal{S}_{1}\left(\begin{array}{cccccc}
0 & 0 & 0 & 1 & 0 & 0 \\
0 & 0 & 0 & 0 & 0 & 0 \\
0 & 0 & 0 & 0 & 0 & 0 \\
0 & 0 & 0 & 0 & 0 & 0 \\
0 & 0 & 0 & 0 & 0 & 1 \\
0 & 0 & 0 & 0 & 0 & 0
\end{array}\right) \theta^{1} \wedge \theta^{2} \wedge \theta^{3}
$$

where $\mathcal{S}_{1}$ is defined by (6). This formula implies that the Fefferman metrics (8) satisfy the Bach equations iff

$$
\mathcal{S}_{1}=0,
$$

or, what is the same,

$$
\mathrm{d} \mathcal{S} \wedge \theta^{2} \wedge \theta^{3} \wedge\left(2 \Omega_{2}+3 \bar{\Omega}_{2}\right) \wedge \bar{\Omega}_{3}=0 .
$$

The only known example of a CR structure with a Fefferman metric satisfying this condition is presented in [22].

5 It is worthwhile noting that for algebraically general metrics, the Baston-Mason conditions (i), (ii) are also sufficient for the conformal Einstein property [1]. 


\section{Second-order ODEs modulo point transformations}

A second-order ODE

$$
\frac{\mathrm{d}^{2} y}{\mathrm{~d} x^{2}}=Q\left(x, y, \frac{\mathrm{d} y}{\mathrm{~d} x}\right)
$$

for a function $\mathbb{R} \ni x \rightarrow y=y(x) \in \mathbb{R}$, can be alternatively written as a system of two first-order ODEs

$$
\frac{\mathrm{d} y}{\mathrm{~d} x}=p, \quad \frac{\mathrm{d} p}{\mathrm{~d} x}=Q(x, y, p)
$$

for two functions $\mathbb{R} \ni x \rightarrow y=y(x) \in \mathbb{R}$ and $\mathbb{R} \ni x \rightarrow p=p(x) \in \mathbb{R}$. This system defines two (contact) 1-forms

$$
\omega^{1}=\mathrm{d} y-p \mathrm{~d} x, \quad \omega^{2}=\mathrm{d} p-Q \mathrm{~d} x,
$$

on a three-dimensional manifold $J^{1}$, the first jet space, parametrized by coordinates $(x, y, p)$. All information about the ODE (15) is encoded in these two forms. For example, any solution to (15) is a curve $\gamma(x)=(x, y(x), p(x)) \subset J^{1}$ on which the forms (16) vanish.

The two contact 1 -forms $\left(\omega^{1}, \omega^{2}\right)$ can be supplemented by

$$
\omega^{3}=\mathrm{d} x,
$$

so that the three 1 -forms $\left(\omega^{1}, \omega^{2}, \omega^{3}\right)$ constitute a basis of 1 -forms on $J^{1}$. This basis will be the basic object of study in the following.

Under the point transformation of variables

$$
y \rightarrow \tilde{y}=\tilde{y}(x, y), \quad x \rightarrow \tilde{x}=\tilde{x}(x, y),
$$

the function $Q=Q\left(x, y, y^{\prime}\right)$ defining the differential equation (15) changes in a rather complicated way. The corresponding change of the basis $\left(\omega^{1}, \omega^{2}, \omega^{3}\right)$ is

$\omega^{1} \rightarrow \tilde{\omega}^{1}=a_{1} \omega^{1}, \quad \omega^{2} \rightarrow \tilde{\omega}^{2}=a_{2} \omega^{2}+a_{3} \omega^{1}, \quad \omega^{3} \rightarrow \tilde{\omega}^{3}=a_{4} \omega^{3}+a_{5} \omega^{1}$, where $a_{1}, a_{2}, a_{3}, a_{4}, a_{5}$ are real functions on $J^{1}$ such that $a_{1} a_{2} a_{4} \neq 0$ on $J^{1}$.

It is now convenient to introduce the following (slightly unusual) notation. The reason for this will eventually become apparent.

Let $i \neq 0$ denote a real number. In addition, let the real 1 -forms $(\lambda, \mu, \bar{\mu})$ be defined by

$$
\lambda=-i \omega^{1}, \quad \mu=\omega^{2}, \quad \bar{\mu}=\omega^{3} .
$$

It follows from the definition of $\left(\omega^{1}, \omega^{2}, \omega^{3}\right)$ that

$$
\begin{aligned}
& \lambda \wedge \mu \wedge \bar{\mu} \neq 0, \\
& \mathrm{~d} \lambda \wedge \lambda \neq 0,
\end{aligned}
$$

and that the forms $(\lambda, \mu, \bar{\mu})$ are given up to transformations

$$
\lambda \rightarrow a \lambda, \quad \mu \rightarrow b \mu+c \lambda, \quad \bar{\mu} \rightarrow \bar{b} \bar{\mu}+\bar{c} \lambda,
$$

with real functions $a, b, \bar{b}, c, \bar{c}$ such that $a b \bar{b} \neq 0$.

Conversely, given a three-dimensional manifold $N$ equipped with three real 1 -forms $(\lambda, \mu, \bar{\mu})$ satisfying (20) and (21) and defined up to transformations (22), we can associate with them a point equivalent class of a second-order ODE as follows. Since $\operatorname{dim} N=3$, we have

$$
\mathrm{d} \lambda \wedge \lambda \wedge \bar{\mu}=0 \quad \text { and } \quad \mathrm{d} \bar{\mu} \wedge \lambda \wedge \bar{\mu}=0 .
$$

Hence the Fröbenius theorem [25] applied to the forms $\lambda, \bar{\mu}$ implies that there exist coordinates $(x, y, z)$ on $N$ such that $\lambda=A \mathrm{~d} x+B \mathrm{~d} y$ and $\bar{\mu}=C \mathrm{~d} x+H \mathrm{~d} y$, where $A, B, C, H$ are 
appropriate functions on $N$. Thus, modulo the freedom (22), the forms $\lambda, \bar{\mu}$ can be transformed to $\lambda=\mathrm{d} y-p \mathrm{~d} x, \bar{\mu}=\mathrm{d} x$, where $p$ is a certain function of coordinates $(x, y, z)$ on $N$. But $0 \neq \mathrm{d} \lambda \wedge \lambda=\mathrm{d} p \wedge \mathrm{d} y \wedge \mathrm{d} x$, so $(x, y, p)$ can be considered a new coordinate system on $N$. In these coordinates the form $\mu$ can be written as $\mu=U \mathrm{~d} x+V \mathrm{~d} y+Z \mathrm{~d} p$ so, by means of transformations, (22) can be reduced to $\mu=\mathrm{d} p-Q \mathrm{~d} x$ with $Q=Q(x, y, p)$ being a certain real function on $N$. Thus, the original forms $(\lambda, \mu, \bar{\mu})$ define a point equivalent class of a second-order ODE $y^{\prime \prime}=Q\left(x, y, y^{\prime}\right)$. The above considerations prove the oneto-one correspondence between second-order ODEs given modulo point transformations and equivalence classes of the triples of real 1-forms $(\lambda, \mu, \bar{\mu})$ on 3 -manifolds satisfying (20) and (21) and given up to (22). This enables us to reformulate an equivalence problem for second-order ODEs modulo point transformations in much the same way as an equivalence problem for nondegenerate three-dimensional CR structures.

Definition 3. Two second-order ODEs, represented by the respective real 1-forms $(\lambda, \mu, \bar{\mu})$ and $\left(\lambda^{\prime}, \mu^{\prime}, \bar{\mu}^{\prime}\right)$, on the respective 3-manifolds $N$ and $N^{\prime}$, are locally point equivalent, iff there exists a local diffeomorphism

$$
\phi: N \rightarrow N^{\prime}
$$

and real functions $a \neq 0, b \neq 0, \bar{b} \neq 0, c, \bar{c}$ on $N$ such that

$$
\phi^{*}\left(\lambda^{\prime}\right)=a \lambda, \quad \phi^{*}\left(\mu^{\prime}\right)=b \mu+c \lambda, \quad \phi^{*}(\bar{\mu})=\bar{b} \mu+\bar{c} \lambda .
$$

This definition, when compared with definition 2, indicates that we can treat the forms $(\lambda, \mu, \bar{\mu})$ representing second-order ODEs as the respective analogues of the forms $(\lambda, \mu, \bar{\mu})$ representing nondegenerate three-dimensional CR structures. It also indicates that the solution for the equivalence problem for second-order ODEs modulo point transformations should be given by a theorem analogous to theorem 1 . Actually, with the above-introduced notation, in which all three 1 -forms $(\lambda, \mu, \bar{\mu})$ are real, $i \neq 0$ is a real number and the 'bar' symbol merely denotes that a given variable (a function, or a form) is totally independent of its nonbarred counterpart, we obtain the solution of the equivalence problem for ODEs by the following reinterpretation of theorem 1. First, given a point equivalence class of second-order ODEs, represented by forms $(\lambda, \mu, \bar{\mu})$, we associate with it the forms

$$
\theta^{1}=b \mu+c \lambda, \quad \theta^{2}=\bar{b} \bar{\mu}+\bar{c} \lambda, \quad \theta^{3}=a \lambda .
$$

Then the analogue of theorem 1 is as follows.

Theorem 2. Every second-order ODE given modulo point transformations uniquely defines an eight-dimensional manifold $P$, real 1 -forms $\theta^{1}, \theta^{2}, \theta^{3}, \Omega_{2}, \bar{\Omega}_{2}, \Omega_{3}, \bar{\Omega}_{3}, \Omega_{4}$ and real functions $\mathcal{R}, \overline{\mathcal{R}}, \mathcal{S}, \overline{\mathcal{S}}$ on $P$ such that

- $\theta^{1}, \theta^{2}, \theta^{3}$ are as in (23)

- $\theta^{1} \wedge \theta^{2} \wedge \theta^{3} \wedge \Omega_{2} \wedge \bar{\Omega}_{2} \wedge \Omega_{3} \wedge \bar{\Omega}_{3} \wedge \Omega_{4} \neq 0$ at each point of $P$.

The forms satisfy the following equations:

$$
\begin{aligned}
& \mathrm{d} \theta^{1}=\Omega_{2} \wedge \theta^{1}+\Omega_{3} \wedge \theta^{3} \\
& \mathrm{~d} \theta^{2}=\bar{\Omega}_{2} \wedge \theta^{2}+\bar{\Omega}_{3} \wedge \theta^{3} \\
& \mathrm{~d} \theta^{3}=i \theta^{1} \wedge \theta^{2}+\left(\Omega_{2}+\bar{\Omega}_{2}\right) \wedge \theta^{3} \\
& \mathrm{~d} \Omega_{2}=2 i \theta^{1} \wedge \bar{\Omega}_{3}+i \theta^{2} \wedge \Omega_{3}+\Omega_{4} \wedge \theta^{3} \\
& \mathrm{~d} \bar{\Omega}_{2}=-2 i \theta^{2} \wedge \Omega_{3}-i \theta^{1} \wedge \bar{\Omega}_{3}+\Omega_{4} \wedge \theta^{3} \\
& \mathrm{~d} \Omega_{3}=\Omega_{4} \wedge \theta^{1}+\Omega_{3} \wedge \bar{\Omega}_{2}+\mathcal{R} \theta^{2} \wedge \theta^{3} \\
& \mathrm{~d} \bar{\Omega}_{3}=\Omega_{4} \wedge \theta^{2}+\bar{\Omega}_{3} \wedge \Omega_{2}+\overline{\mathcal{R}} \theta^{1} \wedge \theta^{3} \\
& \mathrm{~d} \Omega_{4}=i \Omega_{3} \wedge \bar{\Omega}_{3}+\Omega_{4} \wedge\left(\Omega_{2}+\bar{\Omega}_{2}\right)+\overline{\mathcal{S}} \theta^{1} \wedge \theta^{3}+\mathcal{S} \theta^{2} \wedge \theta^{3} .
\end{aligned}
$$


The functions $\mathcal{R}, \overline{\mathcal{R}}, \mathcal{S}, \overline{\mathcal{S}}$, satisfy in addition

$$
\begin{aligned}
& \mathrm{d} \mathcal{R}=-\mathcal{R}\left(\Omega_{2}+3 \bar{\Omega}_{2}\right)-\mathcal{S} \theta^{1}+\mathcal{R}_{2} \theta^{2}+\mathcal{R}_{3} \theta^{3} \\
& \mathrm{~d} \overline{\mathcal{R}}=-\overline{\mathcal{R}}\left(\bar{\Omega}_{2}+3 \Omega_{2}\right)-\overline{\mathcal{S}} \theta^{2}+\overline{\mathcal{R}}_{2} \theta^{1}+\overline{\mathcal{R}}_{3} \theta^{3}
\end{aligned}
$$

and

$$
\begin{aligned}
& \mathrm{d} \mathcal{S}=-\mathcal{S}\left(2 \Omega_{2}+3 \bar{\Omega}_{2}\right)-i \mathcal{R} \bar{\Omega}_{3}+\mathcal{S}_{1} \theta^{1}+\mathcal{S}_{2} \theta^{2}+\mathcal{S}_{3} \theta^{3} \\
& \mathrm{~d} \overline{\mathcal{S}}=-\overline{\mathcal{S}}\left(2 \bar{\Omega}_{2}+3 \Omega_{2}\right)+i \overline{\mathcal{R}} \Omega_{3}+\overline{\mathcal{S}}_{2} \theta^{1}+\mathcal{S}_{1} \theta^{2}+\overline{\mathcal{S}}_{3} \theta^{3},
\end{aligned}
$$

with appropriate functions $\mathcal{R}_{2}, \overline{\mathcal{R}}_{2}, \mathcal{R}_{3}, \overline{\mathcal{R}}_{3}, \mathcal{S}_{1}, \overline{\mathcal{S}}_{1}, \mathcal{S}_{2}, \overline{\mathcal{S}}_{2}, \mathcal{S}_{3}, \overline{\mathcal{S}}_{3}$.

The function $\mathcal{S}_{1}$ satisfies

$$
\mathcal{S}_{1}=\overline{\mathcal{S}}_{1} \text {. }
$$

Given an equation $y^{\prime \prime}=Q\left(x, y, y^{\prime}\right)$ and the standard coordinate system $(x, y, p)$ on $N=J^{1}$, we introduce the vector field

$$
D=\partial_{x}+p \partial_{y}+Q \partial_{p}, \quad Q=Q(x, y, p) .
$$

The coordinates $(x, y, p)$ can be extended to a coordinate system $(x, y, p, \rho, \phi, \gamma, \bar{\gamma}, r)$ on $P$ in which the forms and functions of the above theorem can be written as follows:

$$
\begin{aligned}
& \lambda=-i(\mathrm{~d} y-p \mathrm{~d} x), \quad \mu=\mathrm{d} p-Q \mathrm{~d} x, \quad \bar{\mu}=\mathrm{d} x \\
& \theta^{1}=\rho \mathrm{e}^{\mathrm{i} \phi}(\mu+\gamma \lambda), \quad \theta^{2}=\rho \mathrm{e}^{-\mathrm{i} \phi}(\bar{\mu}+\bar{\gamma} \lambda), \quad \theta^{3}=\rho^{2} \lambda \\
& \Omega_{2}=i \mathrm{~d} \phi+\frac{\mathrm{d} \rho}{\rho}+\frac{1}{4 i \rho^{2}}\left[6 \gamma \bar{\gamma} i^{2}-6 \bar{\gamma} i Q_{p}-Q_{p p}-4 i r \rho\right] \theta^{3}-\frac{2 i \bar{\gamma}}{\rho} \mathrm{e}^{-\mathrm{i} \phi} \theta^{1} \\
& -\frac{\mathrm{e}^{\mathrm{i} \phi}}{\rho}\left(i \gamma-Q_{p}\right) \theta^{2} \\
& \bar{\Omega}_{2}=-i \mathrm{~d} \phi+\frac{\mathrm{d} \rho}{\rho}-\frac{1}{4 i \rho^{2}}\left[6 \gamma \bar{\gamma} i^{2}-2 \bar{\gamma} i Q_{p}-Q_{p p}+4 i r \rho\right] \theta^{3} \\
& +\frac{i \bar{\gamma}}{\rho} \mathrm{e}^{-\mathrm{i} \phi} \theta^{1}+\frac{\mathrm{e}^{\mathrm{i} \phi}}{\rho}\left(2 i \gamma-Q_{p}\right) \theta^{2} \\
& \Omega_{3}=\frac{\mathrm{e}^{\mathrm{i} \phi}}{\rho}\left[\mathrm{d} \gamma-\frac{1}{6 i^{2} \rho^{2}}\left(D Q_{p p}+6 \gamma^{2} \bar{\gamma} i^{3}-6 \gamma \bar{\gamma} i^{2} Q_{p}-3 \gamma i Q_{p p}-4 Q_{p y}-6 \bar{\gamma} i Q_{y}\right) \theta^{3}\right. \\
& \left.+\frac{\mathrm{e}^{-\mathrm{i} \phi}}{4 i \rho}\left(2 \gamma \bar{\gamma} i^{2}-2 \bar{\gamma} i Q_{p}-Q_{p p}-4 i r \rho\right) \theta^{1}+\frac{\mathrm{e}^{\mathrm{i} \phi}}{i \rho}\left(\gamma^{2} i^{2}-\gamma i Q_{p}-Q_{y}\right) \theta^{2}\right] \\
& \bar{\Omega}_{3}=\frac{\mathrm{e}^{-\mathrm{i} \phi}}{\rho}\left[\mathrm{d} \bar{\gamma}+\frac{1}{6 i^{2} \rho^{2}}\left(6 \gamma \bar{\gamma}^{2} i^{3}-6 \bar{\gamma}^{2} i^{2} Q_{p}-3 \bar{\gamma} i Q_{p p}-Q_{p p p}\right) \theta^{3}-\frac{\mathrm{e}^{-\mathrm{i} \phi}}{\rho} \bar{\gamma}^{2} i \theta^{1}\right. \\
& \left.-\frac{\mathrm{e}^{\mathrm{i} \phi}}{4 i \rho}\left(2 \gamma \bar{\gamma} i^{2}-2 \bar{\gamma} i Q_{p}-Q_{p p}+4 i r \rho\right) \theta^{2}\right] \\
& \Omega_{4}=-\frac{i}{2 \rho^{2}} \bar{\gamma} \mathrm{d} \gamma+\frac{1}{2 \rho^{2}}\left(i \gamma-Q_{p}\right) \mathrm{d} \bar{\gamma}-\frac{\mathrm{d} r}{\rho}-\frac{r \mathrm{~d} \rho}{\rho^{2}}+\frac{1}{48 i^{2} \rho^{4}}\left[8 D Q_{p p p}-3 Q_{p p}^{2}+8 Q_{p} Q_{p p p}\right. \\
& -12 Q_{p p y}-12 \gamma i Q_{p p p}+\bar{\gamma}\left(12 i D Q_{p p}-24 i Q_{p y}\right)-12 \gamma \bar{\gamma} i^{2} Q_{p p} \\
& +\bar{\gamma}^{2}\left(24 i^{2} D Q_{p}+12 i^{2} Q_{p}^{2}-48 i^{2} Q_{y}\right)-48 i^{3} \gamma \bar{\gamma}^{2} Q_{p}+36 \gamma^{2} \bar{\gamma}^{2} i^{4} \\
& \left.+48 \bar{\gamma} r \rho i^{2} Q_{p}+48 i^{2} \rho^{2} r^{2}\right] \theta^{3} \\
& -\frac{\mathrm{e}^{\mathrm{i} \phi}}{12 i \rho^{3}}\left[6 \gamma \bar{\gamma}^{2} i^{3}-6 \bar{\gamma}^{2} i^{2} Q_{p}+3 \bar{\gamma} i Q_{p p}-Q_{p p p}-12 \bar{\gamma} i^{2} r \rho\right] \theta^{1}
\end{aligned}
$$




$$
\begin{gathered}
-\frac{\mathrm{e}^{\mathrm{i} \phi}}{12 i \rho^{3}}\left[D Q_{p p}-4 Q_{p y}-3 i \gamma Q_{p p}+6 i \bar{\gamma}\left(D Q_{p}-2 Q_{y}\right)-6 i^{2} \gamma \bar{\gamma} Q_{p}\right. \\
\left.+6 \gamma^{2} \bar{\gamma} i^{3}+12 \gamma i^{2} r \rho\right] \theta^{2}, \\
\mathcal{R}=-\frac{\mathrm{e}^{2 \mathrm{i} \phi}}{6 i^{2} \rho^{4}} w_{1}, \quad \mathcal{S}=-\frac{\mathrm{e}^{\mathrm{i} \phi}}{3 i^{2} \rho^{5}}\left[\partial_{p} w_{1}+i \bar{\gamma} w_{1}\right], \\
\overline{\mathcal{R}}=-\frac{\mathrm{e}^{-2 \mathrm{i} \phi}}{6 i^{2} \rho^{4}} w_{2}, \quad \overline{\mathcal{S}}=-\frac{\mathrm{e}^{-\mathrm{i} \phi}}{3 i^{2} \rho^{5}}\left[D w_{2}+\left(2 Q_{p}-i \gamma\right) w_{2}\right],
\end{gathered}
$$

where we have introduced functions

$$
w_{1}=D^{2} Q_{p p}-4 D Q_{p y}-D Q_{p p} Q_{p}+4 Q_{p} Q_{p y}-3 Q_{p p} Q_{y}+6 Q_{y y}
$$

and

$$
w_{2}=Q_{\text {pppp }},
$$

which are the relative point invariants of the ODE.

Similarly as in the CR case, theorem 2 can be reinterpreted in terms of the language of Cartan connections. It follows that the manifold $P$ of theorem 2 is a Cartan bundle $H \rightarrow P \rightarrow J^{1}$, with $H$ being a five-dimensional parabolic subgroup of $S L(3, \mathbb{R})$ group. The forms $\theta^{1}, \theta^{2}, \theta^{3}, \Omega_{2}, \bar{\Omega}_{2}, \Omega_{3}, \bar{\Omega}_{3}, \Omega_{4}$ of the theorem can be collected into a matrix of 1 -forms

$$
\omega=\left(\begin{array}{ccc}
\frac{1}{3}\left(2 \Omega_{2}+\bar{\Omega}_{2}\right) & i \bar{\Omega}_{3} & -\frac{1}{2} \Omega_{4} \\
\theta^{1} & \frac{1}{3}\left(\bar{\Omega}_{2}-\Omega_{2}\right) & -\frac{1}{2} \Omega_{3} \\
2 \theta^{3} & 2 i \theta^{2} & -\frac{1}{3}\left(2 \bar{\Omega}_{2}+\Omega_{2}\right),
\end{array}\right),
$$

which is now an $\operatorname{sl}(3, \mathbb{R})$-valued Cartan connection on $P$ (all the variables are real!). It follows from equations (24) that the curvature of these connections is

$$
\Omega=\mathrm{d} \omega+\omega \wedge \omega=\left(\begin{array}{ccc}
0 & i \overline{\mathcal{R}} \theta^{1} \wedge \theta^{3} & -\frac{1}{2} \overline{\mathcal{S}} \theta^{1} \wedge \theta^{3}-\frac{1}{2} \mathcal{S} \theta^{2} \wedge \theta^{3} \\
0 & 0 & -\frac{1}{2} \mathcal{R} \theta^{2} \wedge \theta^{3} \\
0 & 0 & 0
\end{array}\right) .
$$

It yields all invariant information about the corresponding point equivalent class of secondorder ODEs. In particular, the ODEs corresponding to flat $(\mathcal{R}=0, \overline{\mathcal{R}}=0, \mathcal{S}=0, \overline{\mathcal{S}}=0$ ) connections are given by the conditions

$$
w_{1}=0, \quad w_{2}=0 .
$$

They are all point equivalent to the flat equation $y^{\prime \prime}=0$. We remark that the vanishing of $\mathcal{R}$ implies vanishing of $\mathcal{S}$. Each of these two conditions is a point-invariant property of the corresponding ODE. However, the other pair of point-invariant conditions $\overline{\mathcal{R}}=0, \overline{\mathcal{S}}=0$ is totally independent. This is the significant difference between the behaviour of CR structures and second-order ODEs. Indeed, in the classification of nondegenerate three-dimensional CR structures, there are only two major branches: either $\mathcal{R}=0$ (in which case the CR structure is locally equivalent to $\mathbf{S}^{3} \subset \mathbb{C}^{2}$ ) or $\mathcal{R} \neq 0$. In the ODE case $\mathcal{R}$ and $\overline{\mathcal{R}}$ are unrelated and we have four main branches, corresponding to (i) $\mathcal{R}=0, \overline{\mathcal{R}}=0$, (ii) $\mathcal{R}=0, \overline{\mathcal{R}} \neq 0$ (ii') $\mathcal{R} \neq 0, \overline{\mathcal{R}}=0$ and (iii) $\mathcal{R} \neq 0, \overline{\mathcal{R}} \neq 0$. It follows that branches (ii) and (ii') are, in a sense, dual to each other. To explain this duality, we need to introduce the Fefferman metric associated with an ODE.

The system (24) defining the invariant forms of theorem 2 has all the qualitative properties of system (4) of theorem 1 . Thus, introducing the basis $X_{1}, X_{2}, X_{3}, Y_{2}, \bar{Y}_{2}, Y_{3}, \bar{Y}_{3}, Y_{4}$ of vector fields, the respective dual of forms $\theta^{1}, \theta^{2}, \theta^{3}, \Omega_{2}, \bar{\Omega}_{2}, \Omega_{3}, \bar{\Omega}_{3}, \Omega_{4}$, we see that the distribution 
spanned by the four vector fields $Z_{I}=\left(Y_{2}+\bar{Y}_{2}, Y_{3}, \bar{Y}_{3}, Y_{4}\right)$ is integrable. Moreover, the bilinear form

$$
G=2 \theta^{1} \theta^{2}+\frac{2}{3 i} \theta^{3}\left(\Omega_{2}-\bar{\Omega}_{2}\right)
$$

which now has signature $(++--0000)$, has all $Z_{I}$ as degenerate directions. This, when compared with the fact that $G$ is preserved up to a scale during the Lie transport along $Z_{I}$, shows that the four-dimensional space $P / \sim$ of leaves of the distribution spanned by $Z_{I}$ is naturally equipped with the conformal class of split signature metrics $\left[g_{F}\right]$, the class to which the bilinear form $G$ naturally descends. We call the metrics

$$
g_{F}=2 \theta^{1} \theta^{2}+\frac{2}{3 i} \theta^{3}\left(\Omega_{2}-\bar{\Omega}_{2}\right)
$$

on $P / \sim$ the Fefferman metrics associated with a point equivalence class of a second-order ODE $y^{\prime \prime}=Q(x, y, y)$.

The metrics $g_{F}$, when written in coordinates $(x, y, p, \rho, \phi, \gamma, \bar{\gamma}, r)$ on $P$, read

$g_{F}=2 \rho^{2}\left[(\mathrm{~d} p-Q \mathrm{~d} x) \mathrm{d} x-(\mathrm{d} y-p \mathrm{~d} x)\left(\frac{2}{3} i \mathrm{~d} \phi+\frac{2}{3} Q_{p} \mathrm{~d} x+\frac{1}{6} Q_{p p}(\mathrm{~d} y-p \mathrm{~d} x)\right)\right]$.

This enables us to (locally) identify the space parametrized by $(x, y, p, \phi)$ with $P / \sim$ and the space parametrized by $(x, y, p, \phi, \rho)$ with the space of all Fefferman metrics associated with a given $y^{\prime \prime}=Q\left(x, y, y^{\prime}\right)$.

Proposition 1. The Fefferman conformal class of metrics $\left[g_{F}\right]$ associated with a point equivalent class of ODEs has the following properties.

- Each $g_{F}$ has signature $(++--)$.

- The Weyl tensor of each $g_{F}$ has both self-dual and anti-self-dual parts of Petrov type N. The self-dual part $C^{+}$is proportional to $\mathcal{R}$ and the anti-self-dual part $C^{-}$is proportional to $\overline{\mathcal{R}}$.

- $g_{F}$ satisfies the Baston-Mason conditions (12) if and only if the corresponding point equivalent class of equations satisfies either $\mathcal{R}=0$ or $\overline{\mathcal{R}}=0$.

The first two statements of the above proposition are obvious in view of formulae (9) and (11). To prove the last statement, we calculate the Baston-Mason conditions (12) in coordinates $(x, y, p, \phi)$. A short calculation and the identity

$$
w_{1 p p}=\left(D^{2}+3 Q_{p} D+2 D Q_{p}+2 Q_{p}^{2}-Q_{y}\right) w_{2}
$$

show that these conditions are equivalent to

$$
\left(i^{\prime}\right) \quad w_{1 p p}=0 \quad \text { and } \quad\left(\text { ii }^{\prime}\right) \quad w_{1} w_{2}=0 \text {, }
$$

where $\left(i^{\prime}\right)$ corresponds to the vanishing of the Yang-Mills current of the Cartan normal conformal connection $\tilde{\omega}$ associated with $g_{F}$ via (9) and (ii') corresponds to the Baston-Mason condition (ii) of (12). Comparison of (32) and (33) proves that the necessary and sufficient condition for (12) is $w_{1}=0$ or $w_{2}=0$. This in particular means that such metrics must be either self-dual or anti-self-dual. 
Remark. Note that one of the principal null directions of $g_{F}$, generated by the vector field $k=\partial_{\phi}$, is a conformal Killing vector field for $g_{F}$. It generates a congruence of null shear-free and twisting geodesics on $P / \sim$. This statement, together with the above proposition 1 , totally characterizes the Fefferman metrics $g_{F}[35]$.

Since metrics $g_{F}$ are algebraically special (of type $N \times N^{\prime}$ or its specializations), the BastonMason conditions (12) are not sufficient to guarantee the conformal Einstein property for them. All the Fefferman metrics which are conformal to Einstein metrics are given in the appendix.

It is very easy to determine all classes of second-order ODEs corresponding to the selfdual Fefferman metrics. These are all equations for which $w_{2}=0$, i.e. all the equations of the form

$$
y^{\prime \prime}=A_{0}(x, y)+A_{1}(x, y) y^{\prime}+A_{2}(x, y)\left(y^{\prime}\right)^{2}+A_{3}(x, y)\left(y^{\prime}\right)^{3},
$$

for which the function $Q$ is an arbitrary polynomial of the third order in the variable $p$. Finding classes of equations corresponding to the anti-self-dual metrics is more difficult but, surprisingly, possible, due to another notion of duality: the duality of second-order ODEs.

Given a second-order ODE of the form

$$
\frac{\mathrm{d}^{2} y}{\mathrm{~d} x^{2}}=Q\left(x, y, \frac{\mathrm{d} y}{\mathrm{~d} x}\right)
$$

consider its general solution $y=y(x, X, Y)$, where $X, Y$ are constants of integration. In the space $\mathbb{R}^{2} \times \mathbb{R}^{2}$ parametrized by $(x, y, X, Y)$, this solution can be considered a threedimensional hypersurface

$$
N=\left\{(x, y, X, Y) \in \mathbb{R}^{2} \times \mathbb{R}^{2} \mid G(x, y, X, Y)=y-y(x, X, Y)=0\right\} .
$$

Assuming that $G(x, y, X, Y)=0$ can be solved with respect to $Y$, one gets a function $Y=Y(X, x, y)$. Treating $x$ and $y$ as constant parameters, we can eliminate them by double differentiation of $Y$ with respect to $X$. This means that $Y=Y(X, x, y)$ can be considered a solution to a second-order ODE

$$
\frac{\mathrm{d}^{2} Y}{\mathrm{~d} X^{2}}=q\left(X, Y, \frac{\mathrm{d} Y}{\mathrm{~d} X}\right) .
$$

In passing from (34) to (35) we have chosen $X$ to be an independent variable and $Y$ to be the dependent one. But other choices are possible. In general, we could have chosen two independent functions $\xi=\xi(X, Y)$ and $\zeta=\zeta(X, Y)$ and have treated $\xi$ and $\zeta$ as independent and dependent variables, respectively. Then, after double differentiation with respect to $\xi$, which eliminates the parameters $(x, y)$, we would see that the function $\zeta$ also satisfied a second-order ODE, which would be quite different from (35). It is, however, obvious that this other second-order ODE would be in the same point equivalence class as (35). Thus, given a point equivalence class of ODEs generated by (34), there is a uniquely defined equivalence class of ODEs (35) associated with it. The class (35) is called a class of the dual equations to the equations from the class (34). The following example shows the usefulness of this concept.

Example. The relative invariants $w_{1}$ and $w_{2}$ calculated for the second-order ODE

$$
y^{\prime \prime}=\frac{a}{y^{3}},
$$


where $a \neq 0$ is a real constant, read

$$
w_{1}=\frac{72 a}{y^{5}} \quad \text { and } \quad w_{2}=0 .
$$

Therefore the Fefferman metrics

$$
g_{F}=2 \rho^{2}\left[\left(\mathrm{~d} p-\frac{a}{y^{3}} \mathrm{~d} x\right) \mathrm{d} x-\frac{2}{3} i(\mathrm{~d} y-p \mathrm{~d} x) \mathrm{d} \phi\right]
$$

associated with the point equivalent class of ODEs generated by (36) are self-dual but not conformally flat ${ }^{6}$.

The general solution $y=y(x, X, Y)$ of (36) depends on two arbitrary constants of integration $(X, Y)$ and satisfies

$$
y^{2}=Y(x-X)^{2}+\frac{a}{Y} .
$$

This generates a hypersurface

$$
N=\left\{(x, y, X, Y) \in \mathbb{R}^{2} \times \mathbb{R}^{2} \mid y^{2}=\frac{a}{Y}+Y(x-X)^{2}\right\}
$$

in $\mathbb{R}^{2} \times \mathbb{R}^{2}$. Now, we will treat equation (38) as an equation for a function $Y=Y(X, x, y)$ of an independent variable $X$ and parametrized by $x$ and $y$. Differentiating (38) with respect to $X$ and keeping $x$ and $y$ constants, we get

$$
0=Y^{\prime}(x-X)^{2}-2 Y(x-X)-a \frac{Y^{\prime}}{Y^{2}}
$$

Solving for $x$ and differentiating once more with respect to $X$, we find that $Y=Y(X)$ satisfies the second-order ODE

$$
Y^{\prime \prime}=-\frac{-Y^{4} Y^{\prime 2}+a Y^{\prime 4}-2 Y^{2} Y^{\prime 2} \sqrt{Y^{4}+a Y^{\prime 2}}}{Y^{5}+Y^{3} \sqrt{Y^{4}+a Y^{\prime 2}}} .
$$

This is an equation which generates the point equivalence class of equations dual to (36). This equation has $w_{2} \neq 0$ for each $a \neq 0$. A direct (but lengthy!) calculation shows that

$$
q=q(X, Y, P)=-\frac{-Y^{4} P^{2}+a P^{4}-2 Y^{2} P^{2} \sqrt{Y^{4}+a P^{2}}}{Y^{5}+Y^{3} \sqrt{Y^{4}+a P^{2}}}
$$

has $w_{1}=0$. This is a general fact known already to Cartan [4]. More formally, we have the following proposition.

Proposition 2. The point equivalence class of dual ODEs to a point equivalence class of second-order ODEs for which $w_{2}=0$ and $w_{1} \neq 0$ has $w_{2} \neq 0$ and $w_{1}=0$.

We have already noted (in the above example) that this proposition enables one to find nontrivial solutions to a quite complicated differential equation $w_{1}=0$. Note also that applying the proposition one can obtain quite nontrivial anti-self-dual metrics from rather dull ones (calculate the Fefferman metrics for $q$ of the example, and compare it with (37)). Finally, note that it follows from the proposition that in the classification scheme of the second-order ODEs modulo point transformations, the classification of the $w_{1}=0$ and $w_{2} \neq 0$ case can be obtained from the classification of the simpler $w_{2}=0$ and $w_{1} \neq 0$ case.

6 These are actually the Sparling-Tod metrics well known in twistor theory [36]. 
In [4] proposition 2 is only briefly mentioned ${ }^{7}$. It could be proved by the following line of argument.

The switch between the dual equations $y^{\prime \prime}=Q\left(x, y, y^{\prime}\right)$ and $Y^{\prime \prime}=q\left(X, Y, Y^{\prime}\right)$ essentially means the switch between the contact forms $\omega^{2}=\mathrm{d} p-Q \mathrm{~d} x$ and $\omega^{3}=\mathrm{d} x$. To see this consider the general solution $y=y(x, X, Y)$ of the original equation. Now, pass from the canonical coordinates $(x, y, p)$ on the first jet bundle $J^{1}$ to the new coordinates $(s, X, Y)$ defined via

$$
x=s, \quad y=y(s, X, Y) \quad \text { and } \quad p=y_{s}(s, X, Y) .
$$

In these new coordinates the contact forms

$$
\omega^{1}=\mathrm{d} y-p \mathrm{~d} x, \quad \omega^{2}=\mathrm{d} p-Q \mathrm{~d} x, \quad \omega^{3}=\mathrm{d} x
$$

associated with the original equation are given by

$$
\omega^{1}=y_{X} \mathrm{~d} X+y_{Y} \mathrm{~d} Y, \quad \omega^{2}=y_{s X} \mathrm{~d} X+y_{s Y} \mathrm{~d} Y, \quad \omega^{3}=\mathrm{d} s .
$$

Thus in the point equivalence class of the forms $\omega^{1}$ and $\omega^{2}$, there are forms

$$
\omega^{1}=\mathrm{d} Y+\frac{y_{X}}{y_{Y}} \mathrm{~d} X \quad \text { and } \quad \omega^{2}=\mathrm{d} X .
$$

Moreover, the condition $0 \neq \mathrm{d} \omega^{1} \wedge \omega^{1}=\mathrm{d}\left(\frac{y_{X}}{y_{Y}}\right) \wedge \mathrm{d} X \wedge \mathrm{d} Y$ implies that the three functions

$$
X, Y \text { and } P=-\frac{y_{X}}{y_{Y}}
$$

constitute a coordinate system on $J^{1}$. Therefore, $s$ must be a function of these three variables: $s=s(X, Y, P)$. This means that in the equivalence class of the form

$$
\omega^{3}=\mathrm{d} s=s_{X} \mathrm{~d} X+s_{Y} \mathrm{~d} Y+s_{P} \mathrm{~d} P,
$$

there is $\omega^{3}$ which can be written as

$$
\omega^{3}=\mathrm{d} P-q(X, Y, P) \mathrm{d} X, \quad \text { where } \quad q=-\frac{s_{X}+s_{Y} P}{s_{P}} .
$$

Summarizing, we are able to introduce a coordinate system $(X, Y, P)$ on the first jet bundle $J^{1}$ in which the point equivalence class of the contact forms associated with the original equation $y^{\prime \prime}=Q\left(x, y, y^{\prime}\right)$ can be written as

$$
\omega^{1}=\mathrm{d} Y-P \mathrm{~d} X, \quad \omega^{2}=\mathrm{d} X, \quad \omega^{3}=\mathrm{d} P-q(X, Y, P) \mathrm{d} X .
$$

But this enables us to interpret the $(X, Y, P)$ coordinates as canonical coordinates for the first jet bundle associated with the contact forms

$$
\omega^{1}=\mathrm{d} Y-P \mathrm{~d} X, \quad \omega^{3}=\mathrm{d} P-q(X, Y, P) \mathrm{d} X, \quad \omega^{2}=\mathrm{d} X
$$

of the differential equation $Y^{\prime \prime}=q\left(X, Y, Y^{\prime}\right)$. Because of the original definitions of $X$ and $Y$, this is clearly the dual equation to $y^{\prime \prime}=Q\left(x, y, y^{\prime}\right)$. Note that this interpretation requires the switch between the forms $\omega^{2}$ and $\omega^{3}$. Note also that this switch is compatible with transformations (18) which treat $\omega^{2}$ and $\omega^{3}$ on an equal footing mixing each of them with $\omega^{1}$ only.

7 For an inexperienced reader, it is rather difficult to find a trace of the proposition in the text of [4]. We are very grateful to Crampin [6] for clarifying this point to us. We also take this opportunity to present his understanding of the last paragraph of Cartan's paper [4]. Due to equations (27), (28) and (29), which represent the transformation properties of the forms $\left(\omega^{1}, \omega^{2}, \omega^{3}\right)$ and the relative invariants $w_{1}$ and $w_{2}$, if both $w_{1}$ and $w_{2}$ are nonvanishing, the following forms are (modulo sign) well defined on $J^{1}$.

$$
\begin{array}{ll}
I_{1}=\left(w_{1} w_{2}\right)^{1 / 4} \omega^{1}, & I_{2}=\left(w_{1} w_{2}\right)^{1 / 2} \omega^{1} \wedge \omega^{2} \wedge \omega^{3} \\
I_{3}=w_{1}^{1 / 8} w_{2}^{5 / 8} \omega^{1} \wedge \omega^{2}, & I_{4}=w_{1}^{5 / 8} w_{2}^{1 / 8} \omega^{1} \wedge \omega^{3} .
\end{array}
$$


Once the switch between $y^{\prime \prime}=Q\left(x, y, y^{\prime}\right)$ and $Y^{\prime \prime}=q\left(X, Y, Y^{\prime}\right)$ is understood from the point of view of the switch between $\omega^{2}$ and $\omega^{3}$, it is easy to see that the passage from a differential equation to its dual changes the role of the invariants $w_{1}$ and $w_{2}$. Indeed, looking at the curvature $\Omega$ of the Cartan connection $\omega$ associated with the equation $y^{\prime \prime}=Q\left(x, y, y^{\prime}\right)$, we see that the invariant $w_{1}$ is associated with the $\omega^{3} \wedge \omega^{1}$ term and the invariant $w_{2}$ is associated with the $\omega^{2} \wedge \omega^{1}$ term. Thus, the switch between $\omega^{2}$ and $\omega^{3}$ caused by the switch between the equation and its dual amounts to the switch of $w_{1}$ and $w_{2}$.

From the geometric point of view, the switch between the mutually dual secondorder ODEs can be understood as a transformation that interchanges two naturally defined congruences of lines on the jet bundle $J^{1}$. This bundle is naturally fibred over $J^{0}$ - the plane parametrized by $(x, y)$. The fibres of $J^{1} \rightarrow J^{0}$ are one dimensional and, in the natural coordinates $(x, y, p)$ on $J^{1}$, can be specified by fixing $x$ and $y$. They generate the first congruence of lines on $J^{1}$. The other congruence is defined by the point equivalence class of the equation $y^{\prime \prime}=Q\left(x, y, y^{\prime}\right)$ in the following way. The equation $y^{\prime \prime}=Q\left(x, y, y^{\prime}\right)$ equips $J^{1}$ with the total differential vector field $X_{\bar{\mu}}=D$. Any other equation from the point equivalence class of $y^{\prime \prime}=Q\left(x, y, y^{\prime}\right)$ defines the total differential that differs from $D$ by a scaling functional factor. Thus the lines tangent to all of these total differentials are well defined on $J^{1}$ and generate the second natural congruence of lines. Each of the above congruences on $J^{1}$ defines a natural direction of vector fields tangent to them but, in the canonical coordinates $(x, y, p)$ on $J^{1}$, only the lines of the first congruence can be defined as lines tangent to a particularly simple vector field $X_{\mu}=\partial_{p}$. The passage from the equation to its dual changes the picture: it switches between $X_{\mu}$ and $X_{\bar{\mu}}$, so that the jet bundle $J^{1}$ is now interpreted as a bundle with one-dimensional fibres tangent to $X_{\bar{\mu}}=\partial_{P}$. The space of such fibres may then be identified with the plane parametrized by $(X, Y)$ and the congruence tangent to $X_{\mu}$ by the congruence tangent to the total differential of the dual equation.

\section{Realification of a three-dimensional CR structure}

The analogy between three-dimensional CR structures and second-order ODEs described in the previous two sections can be used to associate a point equivalent class of second-order ODEs with a three-dimensional CR structure. This may introduce a new insight into general relativity, where the three-dimensional CR structures correspond [37, 39, 40] to congruences of shear-free and null geodesics in spacetimes ${ }^{8}$. In particular, the shear-free congruence of null geodesics associated with the celebrated Kerr spacetime can be interpreted in terms of a certain class of point equivalent second-order ODEs. In this section we provide a framework for this kind of consideration concentrating on three-dimensional CR structures that admit two-dimensional groups of local symmetries ${ }^{9}$.

A three-dimensional CR structure with two symmetries can be locally described by real coordinates $(u, x, y)$ in which a representative of the forms from the class $[(\lambda, \mu)])$ is given by

$$
\lambda=\mathrm{d} u+f(y) \mathrm{d} x, \quad \mu=\mathrm{d} x+\mathrm{i} \mathrm{d} y, \quad \bar{\mu}=\mathrm{d} x-\mathrm{i} \mathrm{d} y .
$$

Here the function $f=f(y)$ is real and $\mathrm{i}^{2}=-1$.

To pass from the above CR structures to the corresponding point equivalence class of second-order ODEs we require that the symbol $i$ is a nonvanishing real constant so that the

8 Such spacetimes, the Lorentzian four-dimensional manifolds admitting a null congruence of shear-free geodesics, are called Robinson manifolds [24, 41, 42] and are known to be the analogues of Hermitian manifolds of fourdimensional Riemannian geometry.

9 Note that the Kerr congruence is in this class of examples [20]. 
forms (39) become real and we can interpret them as the forms that via (22) define a point equivalence class of second-order ODEs. To find a particular representative of on ODE in this class, we introduce new coordinates $(\bar{u}, \bar{x}, \bar{y})$, which are related to $(u, x, y)$ by

$$
u=\bar{u}, \quad y=\bar{y}, \quad x=\bar{x}+i \bar{y} .
$$

Since $i$ is now real and nonvanishing, this is a real transformation of the coordinates. It brings $(\lambda, \mu, \bar{\mu})$ to the form

$$
\lambda=\mathrm{d}\left(\bar{u}+i \int f(\bar{y}) \mathrm{d} \bar{y}\right)+f(\bar{y}) \mathrm{d} \bar{x}, \quad \mu=\mathrm{d} \bar{x}+2 i \mathrm{~d} \bar{y}, \quad \bar{\mu}=\mathrm{d} \bar{x} .
$$

After another coordinate transformation

$$
X=\bar{x}, \quad Y=\bar{u}+i \int f(\bar{y}) \mathrm{d} \bar{y}, \quad P=-f(\bar{y})
$$

and an application of the chain rule, one sees that in the class (22) of 1 -forms $(\lambda, \mu, \bar{\mu})$ there are forms

$$
\lambda=\mathrm{d} Y-P \mathrm{~d} X, \quad \mu=\mathrm{d} P-\left.\frac{1}{2 i} f^{\prime}(y)\right|_{y=f^{-1}(-P)} \mathrm{d} X, \quad \bar{\mu}=\mathrm{d} X .
$$

This means that the point equivalence classes of second-order ODEs associated with the CR structures generated by $f=f(y)$ are represented by the equations of the form

$$
Y^{\prime \prime}=\left.\frac{1}{2 i} f^{\prime}(y)\right|_{y=f^{-1}\left(-Y^{\prime}\right)} \text {. }
$$

Consider, in particular, the family of CR structures which have three-dimensional symmetries of Bianchi type $V I_{k}$ [23]. They are parametrized by the Bianchi type parameter $k<0$ and are represented by the function $f=y^{n}$, where $n=n(k)$ is an appropriate [23] function of $k$. Then the point equivalent class of ODEs associated with each $n$ is given by

$$
Y^{\prime \prime}=\frac{n}{2 i}\left(-Y^{\prime}\right)^{1-\frac{1}{n}}
$$

The application of the above results to the new understanding of the congruences of shearfree and null geodesics in spacetime and, in particular, to the Kerr congruence ${ }^{10}$ may be of some use in general relativity theory. Here we focus on properties of Fefferman metrics associated with equations (40). It is known [22] that the Fefferman metrics associated with the three-dimensional CR structures admitting three symmetries of Bianchi type corresponding to $n=-3$ satisfy the Bach equations. They are the only known Lorentzian metrics of twisting type $N$ which satisfy the Bach equations and which are not conformal to Einstein metrics. Similarly, the Fefferman metrics for the equations point equivalent to equation (40) with $n=-3$ provide an example of split signature metrics of type $N \times N^{\prime}$ which satisfy the Bach equations and which are never conformal to Einstein metrics. Explicitly, these metrics are conformal to

$g_{F}=2\left(i P^{\frac{2}{3}} \mathrm{~d} P+\frac{3}{2} P^{2} \mathrm{~d} X\right) \mathrm{d} X-2(\mathrm{~d} Y-P \mathrm{~d} X)\left(\frac{2}{3} i^{2} P^{\frac{2}{3}} \mathrm{~d} \Phi-\frac{1}{9} \mathrm{~d} Y-\frac{11}{9} P \mathrm{~d} X\right)$.

These metrics can be generalized by considering second-order ODEs point equivalent to

$$
y^{\prime \prime}=h\left(y^{\prime}\right),
$$

where $h=h(p)$ is a sufficiently smooth real function. Then the Fefferman metrics associated with such equations are given by

$g_{F}=2 \rho^{2}\left[(\mathrm{~d} p-h \mathrm{~d} x) \mathrm{d} x-(\mathrm{d} y-p \mathrm{~d} x)\left(\frac{2}{3} i \mathrm{~d} \phi+\frac{2}{3} h^{\prime} \mathrm{d} x+\frac{1}{6} h^{\prime \prime}(\mathrm{d} y-p \mathrm{~d} x)\right)\right]$.

The invariants $w_{1}$ and $w_{2}$ for these metrics are

$$
w_{1}=h^{2} h^{\prime \prime \prime \prime}, \quad w_{2}=h^{\prime \prime \prime \prime},
$$

${ }^{10}$ This congruence may be represented by $f=\frac{1}{\cosh ^{2}(y)}$ [20]. 
so the metrics are not conformal to Einstein metrics iff

$$
h^{\prime \prime \prime \prime} \neq 0 \text {. }
$$

This, together with (33), shows that every solution to the equation

$$
h^{2} h^{\prime \prime \prime \prime}=a p+b
$$

where $a$ and $b$ are real constants such that at least one of them does not vanish defines, via (41), a split signature conformal class of metrics that satisfies the Bach equations, is of type $N \times N^{\prime}$ and is not conformal to Einstein metrics. By an appropriate complexification of these metrics, one may get the generalization of the Lorentzian Bach non-Einstein metrics (45) of [22].

\section{Acknowledgments}

This work is a byproduct of the lectures on 'Cartan's equivalence method' which one of us (PN) delivered at the Department of Physics and Astronomy at Pittsburgh Unversity in the winter of 2002-2003. The topic presented here would never even have been touched by us if Ezra Ted Newman would not have requested to hear such lectures. He, his collaborators and the PhD students of the mathematics and physics and astronomy departments at Pittsburgh University were our first audience and our inspiration.

We are very grateful to Lionel Mason, David Robinson and Andrzej Trautman, who carefully read the draft of this paper suggesting several crucial improvements.

The paper was completed during the stay of one of us at King's College, London. We acknowledge support from EPSRC grant GR/534304/01 while at King's College London, NSF grant PHY-0088951 while at Pittsburgh University and the KBN grant 2 P03B 12724 while at Warsaw University.

\section{Appendix}

In this appendix we find all Fefferman metrics (31) which are conformally equivalent to the Einstein metrics. First, we write them in the form

$$
g_{F}=2 \theta^{1} \theta^{2}+\theta^{3} \theta^{4}
$$

with

$$
\begin{aligned}
\theta^{1} & =\rho(\mathrm{d} p-Q \mathrm{~d} x) \\
\theta^{2} & =\rho \mathrm{d} x \\
\theta^{3} & =-\rho^{2}(\mathrm{~d} y-p \mathrm{~d} x) \\
\theta^{4} & =\frac{2}{3} i \mathrm{~d} \phi+\frac{2}{3} Q_{p} \mathrm{~d} x+\frac{1}{6} Q_{p p}(\mathrm{~d} y-p \mathrm{~d} x),
\end{aligned}
$$

where $\rho=\rho(x, y, p, \phi)$ is a function on $J^{1}$. We search for all $\rho$ and $Q=Q(x, y, p)$ for which the Einstein equations

$$
\operatorname{Ric}\left(g_{F}\right)=\Lambda g_{F}
$$

are satisfied. Assuming that $g_{F}$ obey equations (43) we use the implied Weyl tensor identity $\nabla_{\mu} C^{\mu}{ }_{\nu \rho \sigma}=0$. Its $\{113\}$ and $\{223\}$ components imply the equations

$$
\left(3 \rho_{\phi}-i \rho\right) w_{2}=0 \quad \text { and } \quad\left(3 \rho_{\phi}+i \rho\right) w_{1}=0 .
$$

Thus, the metrics $g_{F}$ may be conformal to Einstein metrics only in the following three cases:

$$
\begin{array}{ll}
(\bullet) & w_{1}=w_{2}=0 \\
(\bullet \bullet) & w_{2}=0, w_{1} \neq 0 \text { and } \rho=A(x, y, p) \exp \left(-\frac{\mathrm{i} \phi}{3}\right) \\
(\bullet \bullet \bullet) & w_{2} \neq 0, w_{1}=0 \text { and } \rho=A(x, y, p) \exp \left(\frac{\mathrm{i} \phi}{3}\right) .
\end{array}
$$


Case $(\bullet)$ corresponds to ODEs with flat Cartan connection. These have conformally flat Fefferman metrics.

In the $(\bullet \bullet)$ case, the general solution for the Einstein equations (43) is given by (42), where either

$$
\rho=\frac{\exp \left(-\frac{2}{3} a\right)}{p+2 b} \exp \left(-\frac{\mathrm{i} \phi}{3}\right)
$$

and

$$
\begin{gathered}
Q=p^{3} c+p^{2}\left(6 b c-2 a_{y}\right)+p\left(12 b^{2} c-3 b_{y}-6 b a_{y}-a_{x}\right) \\
+8 b^{3} c-2 b b_{y}-4 b^{2} a_{y}-2 b_{x}-2 b a_{x}
\end{gathered}
$$

or

$$
\rho=\exp \left(-\frac{2}{3} a\right) \exp \left(-\frac{\mathrm{i} \phi}{3}\right)
$$

and

$$
Q=p^{2} a_{y}+2 p a_{x}+b,
$$

with $a=a(x, y), b=b(x, y), c=c(x, y)$ being arbitrary functions of variables $x, y$. All these solutions are Ricci flat. They exhaust the list of Fefferman metrics which have $w_{2}=0$ and are conformal to non-flat Einstein metrics. Since $Q$ in these solutions depends only on at most three arbitrary functions of two variables and generic $Q$ for which $w_{2}=0$ depends on four functions of two variables, then not all ODEs with $w_{2}=0$ have Fefferman metrics which are conformal to Einstein metrics.

The Einstein equations in the $(\bullet \bullet \bullet)$ case reduce to

$$
\rho=a \exp \left(\frac{\mathrm{i} \phi}{3}\right)
$$

where the function $a=a(x, y)$ satisfies a single differential equation

$$
36(D a)^{2}+6 a\left(-3 D^{2} a+Q_{p} D a\right)+a^{2}\left(6 D Q_{p}-18 Q_{y}-4 Q_{p}^{2}\right)=0 .
$$

It follows, as it should, that one of the integrability conditions for this equation is $w_{1}=0$. It is not clear what other conditions should be imposed on $Q$ to guarantee the solvability of (50). If, for example, $Q$ satisfies

$$
6 D Q_{p}-18 Q_{y}-4 Q_{p}^{2}=0
$$

a simple solution is given by $a=$ const.

Finally, we remark that all the metrics $g_{F}$ which, via (42), correspond to solutions (49),

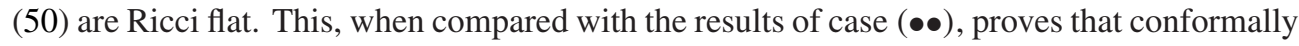
nonflat Fefferman-Einstein metrics have vanishing cosmological constant.

\section{References}

[1] Baston R J and Mason L J 1987 Conformal gravity, the Einstein equations and spaces of complex null geodesics Class. Quantum Grav. 4 815-26

[2] Burns D Jr, Diederich K and Schnider S 1977 Distinguished curves in pseudoconvex boundaries Duke Math. J. 44 407-31

[3] Cartan E 1921 Sur les espaces conformes generalises et l'universe optique C. R. Acad. Sci. Paris 174 857-9

[4] Cartan E 1924 Varietes a connexion projective Bull. Soc. Math. LII 205-41

[5] Cartan E 1932 Sur la geometrie pseudo-conforme des hypersurfaces de l'espace de deux variables complexes. Part I Ann. Math. Pura Appl. 11 17-90

Cartan E 1932 Part II Ann. Scuola Norm. Sup. Pisa 1333-54 
[6] Crampin M 2003 Cartan's concept of duality for second-order differential equations in preparation

[7] Fefferman C L 1976 Monge-Ampere equations, the Bergman kernel, and geometry of pseudoconvex domains Ann. Math. 103 395-416

Fefferman C L 1976 Ann. Math. 104 393-94 (erratum)

[8] Fritelli S, Kozameh C, Newman E T and Nurowski P 2002 Cartan normal conformal connections from differential equations Class. Quantum Grav. $195235-47$

[9] Graham C R 1987 On Sparling's characterization of Fefferman metrics Am. J. Math. 109 853-74

[10] Hitchin N 1982 Complex manifolds and Einstein's equations Twistor Geometry and Non-linear Systems (Springer Lecture Notes in Mathematics 970) ed H D Doebner et al (New York: Springer)

[11] Jacobowitz H 1990 An Introduction to CR Structures (Providence, RI: American Mathematical Society)

[12] Kobayashi S 1972 Transformation Groups in Differential Geometry (Berlin: Springer)

[13] Kozameh C, Newman E T and Nurowski P 2003 Conformal Einstein equations and Cartan conformal connections Class. Quantum Grav. 20 3029-35

[14] Lewandowski J 1988 On the Fefferman class of metrics associated with 3-dimensional CR space Lett. Math. Phys. 15 129-35

[15] Lewandowski J and Nurowski P 1990 Algebraically special twisting gravitational fields and three-dimensional CR structures Class. Quantum Grav. 7 309-28

[16] Lewandowski J, Nurowski P and Tafel J 1990 Einstein's equations and realizability of CR manifolds Class. Quantum Grav. 7 L241-6

[17] Lewandowski J, Nurowski P and Tafel J 1991 Algebraically special solutions of the Einstein equations with pure radiation fields Class. Quantum Grav. 8 493-501

[18] Lie S 1924 Klassifikation und Integration von gewohnlichen Differentialgleichungen zwischen $x$, $y$, die eine Gruppe von Transformationen gestatten III Gesammelte Abhandlungen vol 5 (Leipzig: Teubner)

[19] Newman E T and Nurowski P 2003 Projective connections associated with second-order ODEs Class. Quantum Grav. 20 2325-35

[20] Nurowski P 1993 Einstein equations and Cauchy-Riemann geometry PhD Thesis SISSA

[21] Nurowski P 2003 Notes on Cartan connections, in preparation

[22] Nurowski P and Plebanski J F 2001 Non vacuum twisting type N-metrics Class. Quantum Grav. 18 $341-51$

[23] Nurowski P and Tafel J 1988 Symmetries of CR spaces Lett. Math. Phys. 15 31-8

[24] Nurowski P and Trautman A 2002 Robinson manifolds as the Lorentzian analogs of Hermite manifolds Diff. Geom. Appl. 17 175-95

[25] Olver P J 1996 Equivalence Invariants and Symmetry (Cambridge: Cambridge University Press)

[26] Penrose R 1960 A spinor approach to general relativity Ann. Phys., NY 10 171-201

[27] Penrose R 1967 Twistor algebra J. Math. Phys. 8 345-66

[28] Penrose R 1983 Physical spacetimes and nonrealizable CR structures Bull. Am. Math. Soc. 8 427-48

[29] Petrov A Z 1954 Classification of spaces defining gravitational fields Sci. Not. Kazan State Univ. 114 55-69

[30] Poincaré H 1907 Les fonctions annalytiques de deux varables et la representation conforme Rend. Circ. Mat. Palermo 23 185-220

[31] Robinson I 1961 Null electromagnetic fields J. Math. Phys. 2 290-1

[32] Robinson I and Trautman A 1986 Cauchy-Riemann structures in optical geometry Proc. 4th Marcel Grossman Meeting in GR ed R Ruffini (Amsterdam: Elsevier)

[33] Robinson I and Trautman A 1989 Optical geometry New Theories in Physics ed Z Ajduk et al (Singapore: World Scientific)

[34] Segre B 1931 Intorno al problema di Poincare della representazione pseudo-conforme Rend. Acc. Lincei 13I 676-83

[35] Sparling G A J 1985 Twistor theory and characterization of Fefferman's conformal structure, unpublished

[36] Sparling G A J and Tod K P 1981 An example of an H-space J. Math. Phys. 22 331-2

[37] Tafel J 1985 On the Robinson theorem and shearfree geodesic null congruences Lett. Math. Phys. 10 33-9

[38] Tafel J, Nurowski P and Lewandowski J 1991 Pure radiation field solutions of the Einstein equations Class. Quantum Grav. 8 L83-8

[39] Trautman A 1984 Deformations of the Hodge map and optical geometry J. Geom. Phys. 1 85-95

[40] Trautman A 1998 On complex structures in physics On Einstein's path. Essays in honor of Engelbert Schucking ed A Harvey (Berlin: Springer) pp 487-501

[41] Trautman A 2002 Robinson manifolds and Cauchy-Riemann spaces Class. Quantum Grav. 19 R1-R10

[42] Trautman A 2002 Robinson manifolds and the shear-free condition Int. J. Mod. Phys. A 17 2735-7

[43] Tresse M A 1894 Sur les invariants differentiels des groupes continus de transformations Acta Math. 18 1-88

[44] Tresse M A 1896 Determinations des invariants ponctuels de l'equation differentielle ordinaire du second ordre $y^{\prime \prime}=\omega\left(x, y, y^{\prime}\right)$ (Leipzig: Hirzel) 